Original Article

\title{
Differences in dual task paradigms and executive function ability for recreational athletes in United Arab Emirates
}

\author{
Deema Bitar, BSc, MSc1)*, Lori Maria Walton, PhD, DPT, MSPT, MPH(s), CLT $^{2}$, \\ Bassima Schbley, PhD ${ }^{3)}$, Maha Ehab Mohamed, BSc ${ }^{1)}$, Mennatallah Adel, BSc, MSc ${ }^{1)}$ \\ 1) Department of Physiotherapy, University of Sharjah: Sharjah, Non-US 00000, United Arab Emirates \\ 2) Department of Physiotherapy, University of Scranton, USA \\ 3) Social Work, Washburn University, USA
}

\begin{abstract}
Purpose] The purpose of this study to measure four components of executive function: (1) cognitive flexibility, (2) inhibition, (3) working memory and (4) processing speed, along with the ability to dual task in recreational athletes. [Participants and Methods] This was a cross-sectional study of $(\mathrm{n}=102)$ male and female participants, between the ages of 18-40 years of age across different levels and types of sport related physical activity. The International Physical Activity Questionnaire (IPAQ), short version, Dual Task Abilities (DTA) were measured utilizing a quantitative, dual task, gait test and Executive Function (EF) was measured through Stroop Color Word Test and Trail Making Test. [Results] Differences in EF and Dual Task-Interference (DTI) in recreational athletes did not show a significant difference between varying types of sport and level of sport related activity, with reported values high across all groups. Males reported better dual task interference abilities than females, though there were no significant differences in executive function between males and females. Executive function performance was the highest among the age group (18-24 years) population, but there were no significant differences between those in the higher age groups (25-34 years) and (35-40 years). [Conclusion] Overall, those participating in the study exhibited high prevalence of strong EF ability, regardless of sport activity type or level. This may suggest that type and level of sport activity may not be important when considering executive function performance maintenance for recreational athletes.

Key words: Executive function, Dual task interference, Recreational athletes
\end{abstract}

(This article was submitted Apr. 22, 2020, and was accepted Aug. 4, 2020)

\section{INTRODUCTION}

Executive functions (EF) are a set of higher-order processes that govern goal-directed action and adaptive responses to novel, complex, or ambiguous situations ${ }^{1)}$. EF include: (1) neurological inhibitory control, (2) working memory (WM), and (3) attentional flexibility. They direct and organize behavioral tactics designed to solve adaptive problems ${ }^{2)}$ and are classified into three main core components: (1) Inhibition [inhibitory control, including self-control (behavioral inhibition) and interference control (selective attention and cognitive inhibition)], (2) WM, and (3) cognitive flexibility (CF) (also called mental flexibility). Furthermore, EFs have a primary role in reasoning, problem solving, and planning ${ }^{3)}$. CF, a primary EF emerges during the preschool period and plays a vital role in performance of complex activities such as sport related activity $^{4}$. CF is also referred to as "shifting or switching" abilities, and allows the individual to alternate between two or more representations, tasks, strategies or behaviors in an adaptive manner ${ }^{5)}$. The concept of EFs began by developing a

*Corresponding Author. Deema Bitar (E-mail: Deema.bitar96@gmail.com)

(C2020 The Society of Physical Therapy Science. Published by IPEC Inc.

(c) (i) $(-)$ This is an open-access article distributed under the terms of the Creative Commons Attribution Non-Commercial No Deriva-

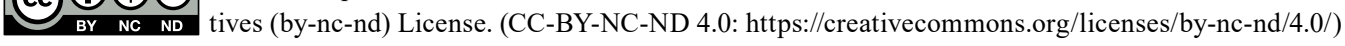


model for a "supervisory attentional system". The "supervisory attentional system" was proposed to refine non-routine, goal-oriented behavior by controlling the selection and maintenance of the goal-relevant task schema'). The central EF is considered the main and global objective for this model. The second step in development of the model focuses on specific functions and processes that require executive attentional control. Four main functions have been proposed, including: (1) coordination in simultaneous tasks as in dual task paradigms, (2) switching between retrieval strategies, (3) focusing on one stimulus and ignoring irrelevant ones, and (4) manipulation of information from long term memory. Baddeley, along with neuropsychological literature identified three components that play an integral role in "self-regulation", known as shifting, updating and inhibition ${ }^{7)}$. These components fall under the term" Metacognitive executive functions" that organize and monitor goal-directed behavior. These functions include abilities assessed by traditional clinical and laboratory measures of $\mathrm{EF}^{8)}$. Shifting is defined as the ability to alternate behaviors between different tasks. Hence, its associated with recognizing contexts and developing behavior appropriate to those contexts. On the other hand, planning is a good example of refining tasks, as it requires monitoring and updating recent information to stored information in the WM. Inhibition is essential for "goal directed behavior" as it prevents inappropriate responses 9 ).

A correlational study was conducted on five and seven-year-old children examining the relationship between educational achievement and contribution to educational achievement expounded the relationship between EF (particularly WM and inhibitory control) and Metacognitive EF. The study reported a chain of significant relationships between EF and metacognitive skills and EF and educational achievement. EF led to the optimal use of metacognitive skills, which in turn led to higher educational achievement in children ${ }^{10)}$. Additional EF are an integral part of neuroscience and psychology and are a well-known foundation in neuroscience and psychology forming from the prefrontal cortex ${ }^{11)}$. In conjunction with EF, the ability to execute two functions at the same time, "Dual Task" (DT), is a neurologic functional term studied extensively in healthy older adult populations ${ }^{12}$. The effects on performance of DT behaviors, known as the change in performance from single to DT, is indicative of 'cognitive-motor' interference ${ }^{16)}$. This may be further explained by a meta-analysis of type and complexity of secondary tasks. DT examines divided attention, which is one subset aspect of EF. Change in task is challenging because of the demand for divided attention, while simultaneously performing two tasks ${ }^{17)}$. However, not all tasks require the same amount of attention due to differences in demand for utilization of EF during performance of each task. For example, interference during walking varies, according to one study that showed tasks that involve internal interference factors (e.g. mathematic tasks) seem to disturb gait performance more than those involving external interfering factors (e.g. reaction time tasks $)^{18)}$.

Furthermore, these tasks were classified according to their task domain, with categories distinguished at the cognitive level ${ }^{17)}$. They were classified as reaction time tasks, discrimination and decision-making tasks, mental tracking tasks and verbal fluency tasks. Reaction time was defined, further, as tasks that involve measuring the elapsed time between a sensory stimulus and a behavioral response ${ }^{17)}$. In addition, discrimination and decision-making tasks were defined as tasks that require selective attention and response to a specific stimulus or feature. Mental Tracking Tasks (MTT) refer to tasks that require holding information in the mind while carrying out a mental process ${ }^{17}$. Verbal Fluency Tasks (VFT) refer to tasks that require spontaneous word production under pre-specified search conditions. Finally, Manual Tasks (MT) refer to balancing tasks of one or both arms, such as cup- or tray-taking tasks ${ }^{19)}$. EF varies from one individual to another and could be influenced by many factors. One research study revealed that physical activity (PA), in adolescence, may have different effects on cognitive performance in adulthood, depending on gender. Adult males who reported higher PA levels in adolescence, demonstrated better performance on EF tests, WM and strategic memory control than peers who had previously been less active. However, no such relationship was found for female ${ }^{20)}$. The finding that EF benefit from PA during the adolescent years fits with the fact that the frontal lobes are still actively developing throughout adolescence, as is reflected by changes in brain tissue, particularly increases in white matter in the pre-frontal corte ${ }^{20)}$. PA refers to any muscular movement requiring substantial energy expenditure and has various subcategories (e.g., leisure activities or exercise). Of these, exercise is exclusively characterized by the intention to develop physical fitness ${ }^{21,22}$. Over the years, previous researches have been addressing the correlation between PA levels and general cognitive functions, these studies found that exercise induced increase in cerebral blood flow (CBF) including the prefrontal cortex, which is commonly associated with EF, thus facilitating the oxygenation of brain areas relevant to cognitive functions ${ }^{23)}$.In several studies, PA was found to lead to releases of neurotrophins in humans promoting the efficiency in neuronal processes ${ }^{24)}$. The purpose of this study is to examine the differences between sport activity levels and type of sport with respect to EF skills and ability of adults to DT for a group of Recreational Athletes (RA) living in the United Arab Emirates (UAE).

Cognitive demands are inherent in many forms of $\mathrm{PA}^{25)}$, as PA encourages cognitive engagement that engages higher order cognitive processes and primes them for subsequent utilization ${ }^{25}$. Activities that require group games or bilateral coordination exercises (order cognitive processing) enhance cognitive functioning more than repetitive and nonadaptive PA of equal intensity and length ${ }^{26)}$. One study compared the effect of short-term intervention and long-term intervention on EF showed that PA can be one approach to improve EF. However, the same study also noted that both interventions had a positive impact on EF, but only long-term interventions were shown to improve all aspects of $\mathrm{EF}^{27}$. A model of "Neurovisceral Integration" proposes anatomical connections between the central and the autonomic nervous system which explains the long-term effect of long-term interventions on physiological learning with EF. According to this model, there is an interaction between parasympathetic cardiac autonomic regulation and EF processes that are controlled by the prefrontal cortex (PFC). This 
network can be considered an internal regulation system through which the brain controls visceromotor, neuroendocrine, and behavioral responses to environmental challenges ${ }^{28}$. Several studies indicate that children and adults, who participate in team sports, tend to adopt a sense of belonging and responsibility toward their community, improvement in their interpersonal and intrapersonal skills, including better verbal and nonverbal communication, and development of self-awareness for understanding their own strengths and weaknesses in addition to the ability to analyze theories and ideas ${ }^{13)}$. Research studies have concluded that team sports help develop children and adults' complex skills required for alerting, orienting and executive attention, particularly among highly competitive and skilled teams ${ }^{14)}$. Furthermore, whether people choose to play sports in teams or solo, psychological factors that have been attributed to improvement in higher EF include a better self-awareness, reduced anxiety, increase in confidence level, and increased motivation ${ }^{15)}$. To date, there is no research that investigates the effects of differing levels of sport activity on EF, specifically the ability to perform DT for RA. For the purpose of our study, RA are defined as people who are involved in sport activity or intense PA for the purpose of leisure, being fit or socializing, at least one time per week.

No research has addressed EF in conjunction with DT for RA populations, thus far and no studies have been done in the UAE. This study fulfills the gap by examining EF from a wider perspective by comparing RA related PA levels for adults in a variety of sports and to measure three components of EF (CF, inhibition, WM and processing speed), along with the individual's ability to DT using a valid and reliable measure for a sample of RA living in the UAE.

\section{PARTICIPANTS AND METHODS}

This study was a cross-sectional, quantitative, quasi-experimental design aimed at examining varying levels of sport. RA, between 18-40 years of age, residing in UAE for at least six months were recruited to volunteer to participate in the study. Exclusion criteria for the study included: disorders that affect EF, such as, hypertension, hepatic encephalopathy, cognitive impairment, Parkinson's Disease, Upper Motor Neuron Disorders, chronic kidney disease, Rheumatoid Arthritis and any psychiatric disorders. In addition to medications that would influence their performance on EF tests.

Sport type was classified as "self-paced" (SP) OR "externally-paced" (EP) sport activity. Self paced (SP) activities included activities such as: walking, weight lifting, CrossFit, and swimming, where the individual's speed and skill determines the performance. EP were defined as sports that require the athlete to react to continuously changing situations and an externally paced environment ${ }^{26}$. Externally paced (EP) activities were defined as those activities that depend on external factors not related to the performer as part of a team and require a reaction to the environment, including activities such as: football, basketball, cricket and badminton. RA were recruited using purposive sampling from Sharjah, Ajman and Abu Dhabi Emirates. Ethical approval was obtained from the Research Ethics Committee of University of Sharjah prior to the initiation of the study and all data collection. The International Physical Activity Questions (IPAQ) is a self-administered questionnaire that includes nine items and is used to assess the intensity level in the Participants' daily activities in MET-min/week ${ }^{29}$.

It covers the three main sports related PA levels Low intensity, Moderate intensity and Vigorous intensity. The Questionnaire's English version test-retest reliability indicates good stability and strong reliability $(\alpha<0.80)^{30)}$. IPAQ short version has also reported a criterion validity median of approximately 0.30 for monitoring population levels of PA among 18 - to 65 -year-old adults in a variety of settings ${ }^{31)}$. Trail making test (TMT) is a neurophysiological test that measures overlapping EF. The test has two parts Part (A) which mainly measure Processing speed ${ }^{32)}$ and part B which measures overlapping EF ${ }^{33)}$. Condition A includes drawing a line connecting 25 circled numbers in ordered sequence $(1,2,3-25)$ as fast as they can. While in condition B they were asked to draw a line alternating between the number and the alphabet in order 13 numbers and letters from A to L (1a, 2b, ,). Time was recorded for both conditions. The test has an $[$ ICC $]=0.742-0.836)^{34)}$. Stroop color word test (SCWT) is used to assess the ability to inhibit cognitive interference that occurs when the processing of a specific stimulus feature impedes the simultaneous processing of a second stimulus attribute ${ }^{35}$. The test includes three main parts. These are; part (A) A4 sheet of color words printed in black ink, part (B) a colored words sheet, and part (c) where words are printed in different ink colors which is the interference trial, where color words are printed in a different ink color that doesn't match (i.e., the word red is printed in yellow). In other words, the Subjects will perform a less automated task (i.e., naming ink color) while inhibiting the interference arising from a more automated task (i.e., reading the word) ${ }^{35}$ ). The outcome of this test was the time to complete Part $\mathrm{C}$ and number of errors, the interference trial, because it requires more attention and response inhibition. Dual task interference (DTI) reflects the relative cost of dual-task performance compared to single-task performance and is expressed as a percentage of single-task performance: DTC $=[(\mathrm{DT}-\text { single task }) /(\text { single task }) \times 100]^{36)}$. Functional gait test: Gait speed which is a gait parameter is assessed during single tasks and DT conditions. Participants are asked to walk for $20 \mathrm{~m}$. For the secondary tasks, one of American Congress of Rehabilitation Medicines' secondary tasks criteria was chosen which was walking with turns and counting backwards in $3 \mathrm{~s}$ which is a cognitive task ${ }^{37)}$. The outcome measure is comparing the speed in DT conditions and single task conditions which was calculated using Excel. Second outcome measure is DTI measured using the traditional formula for evaluating the DTE on a particular outcome of interest ${ }^{38)}$ :

DTE $(\%)=($ DT gait speed - single task gait speed $) /$ single task gait speed $\left.\times 100 \%{ }^{39}\right)$

Negative percentage was defined as "worsened performance" in DT conditions while a positive percentage reflected "better performance" in DT conditions. DT paradigm is an ecologically valid approach for the assessment of cognitive function in conjunction with motor demands ${ }^{40)}$. Data collection was carried out by a licensed physiotherapist and a senior PT 
student under the direction of the primary investigator. Tests were practiced several times by research co-authors to ensure consistency of results. Participation information sheets were distributed to the volunteers to keep for their reference and for future inquiries, along with a copy of the consent form. Volunteers who chose to participate in the study voluntarily signed an informed consent before enrolling them in the study. Volunteers were contacted directly in order to explain the procedure and take into their consent after explaining that the participation is completely voluntary, and they were given the option to withdraw at any time during the research process. Volunteers were introduced to the topic and the details of the procedure by the research co-authors. Participants were approached before starting the exercise to prevent the effect of dose-response relationship between exercise duration and $\mathrm{EF}$ on the results ${ }^{41)}$. The detailed procedure was explained to all participants and questions or clarifications were allowed prior to beginning the activity. Participants were asked to fill out a general health questionnaire and the International Physical Activity Questionnaire- SF (IPAQ-SF) in their language of choice. After filling the questionnaires, participants completed the tests for EF in a private, quiet room to prevent any distractors and ensure confidentiality and privacy. For each volunteer in the study, data collection included their age, occupation, and general health information. All papers and records were kept confidential in the primary investigator's office, locked cabinet. The test outcome was calculated manually in MET-min/week, then were referred back to the IPAQ scoring protocol to get the activity level. The TMT test was administered. Both co-authors were there to explain the test procedure for the volunteers. They were asked to match the numbered circles from 1 to 25 as fast as they can. They were instructed to correct themselves if they made any mistake. The time was recorded using an iPhone 7 stopwatch by both co-authors. Before starting with the tests, there was a sample for both parts A and B that included 4 numbers for part A and 4 numbers and 4 letters for part B. They were instructed to try the sample first before starting the test.

Timer started as soon as the participants placed the pen on number one and stopped when they lifted the pen up. The SCWT was administered on paper, part 1 was printed in black, while 2 and 3 were printed in colors. Paper was $29.70 \mathrm{~cm}$ $\times 20.99 \mathrm{~cm}$. In each part there were 100 words in columns. Two chairs were placed with a distance of $10 \mathrm{~m}$. Distance was measured using a measuring tape. They were instructed to walk at their preferred speed back and forth starting from the chair to the other one turn and walk back total of $(20 \mathrm{~m})$. Then volunteers were asked to take a seat and perform the cognitive task while sitting for $10 \mathrm{sec}$ which was counting backwards in $3 \mathrm{~s}$. At the end, they were asked to walk with their preferred speed and do both tasks as accurately as they can. A pilot study was performed for DTE using SPSS.24 $(n=5$, ICC $=0.992, p=0.001)$. Data was analyzed using SPSS.24.0 (Table 1).

\section{RESULTS}

In total, 102 Participants (65 Male/37 Female) were recruited of different sport related PA levels (13 Low/20 Moderate/69 Vigorous) The instrument's protocol considered walking as 3.3 MET, moderate intensity PA as 4 METS and vigorous intensity PA as 8 METS. To calculate MET-min/week, time was converted into minutes and then MET values were multiplied by time in minutes and number of days per week that activity was performed. Then they were grouped into the three intensity levels using the IPAQ instrument protocol ${ }^{57}$. Mean age was $24.73 \pm 6.63$ years. 14 sport types were reported using the general health questionnaire. Two participants withdrew from the research because they couldn't complete the tests; their data was discarded, while 5 participants had the following disorders and had to be excluded, Multiple sclerosis, attention deficit hyperactivity Disorder (ADHD) and hypertension. Sports type was classified as self-paced (SP) $n=70$ and externally paced (EP) $n=32$ sports. Low sport related PA levels included $n=9$ SP and $n=4$ EP RA, while moderate intensity included $n=14$ SP $n=6$ EP. The majority of our sample were under the vigorous category $n=47$ for SP, $n=22$ for EP. 51 were males and 18 were female RA. The speed of performing part A and B of TMT was recorded in seconds and the mean TMT $-\mathrm{A} 20.52 \pm$ 8.21 seconds, part B $50.49 \pm 17.85$ seconds. The participants' scores were compared to the average scores of TMT part A 29 seconds, part B 70 seconds ${ }^{58)}$.

The time to complete SCWT and number of errors were analyzed for part C. The mean test score for part C in SCWT in

Table 1. Pilot study Intraclass correlation values for Dual Task Test and Single Task Test

\begin{tabular}{lcccc}
\hline & Intraclass & \multicolumn{2}{c}{$95 \% \mathrm{CI}$} & \multirow{2}{*}{ Significance } \\
\cline { 3 - 4 } correlation $^{\mathrm{b}}$ & & Lower bound & Upper bound & \\
\hline Single measure $^{1}$ & $0.843^{\mathrm{a}}$ & -0.045 & 0.983 & $0.003^{* *}$ \\
Average measures $^{2}$ & $0.915^{\mathrm{c}}$ & -0.094 & 0.992 & $0.003^{* *}$ \\
\hline
\end{tabular}

A pilot study was performed to determine inter-rater and test re-test reliability for recording STS and DTS using SPSS.24.

These findings suggest that good inter-tester and test-retest reliability can be achieved for the Melbourne Assessment when used in a group of South African Black children.

These findings suggest good inter-tester and test-retest reliability in testing STS and DTS in recreational athletes. $(\mathrm{n}=5, \mathrm{ICC}=0.992, * * \mathrm{p}=0.003),{ }^{\text {a }}$ The estimator is the same, whether the interaction effect is present or not. ${ }^{b}$ Intraclass correlation coefficient. ${ }^{\mathrm{c}}$ This estimate is computed assuming the interaction effect is absent because it is not estimable otherwise. 
seconds was $101.46 \pm 28.5$, mean errors were $2.8 \pm 3.6$. All these scores are indicative of good processing speed, overlapping $\mathrm{EF}$ and inhibition. The mean DT speed for RA in $\mathrm{m} / \mathrm{s}$ was $1.56 \pm 0.52$, ST $2.04 \pm 0.47$. For DTE the mean was $-23.83 \pm 18.07$. The negative results reflect worsened performance in DT conditions. Only $8.9 \%$ of the sample reported a positive outcome when calculating the DTE. Mean values for TMT-A for SP $(21.13 \pm 8.47)$, EP sports $(19.21 \pm 7.59)$. Mean values for part B were SP (52.5 \pm 19.09$)$, EP (46.09 \pm 14.09$)$. SCWT3 values were in seconds for SP $(103 \pm 29.4)$, EP $(98.05 \pm 26.52)$. DTE for SP had a mean of $(-26.14 \pm 18.71)$, EP $(-18.75 \pm 15.7)$. Independent t-test was done to see the differences between EP and SP sports, the results showed no significant difference between the groups $(\mathrm{p}>0.05)$.

Table 2 shows the mean values for the tests. Kruskal Wallis test was performed to see the differences between the 3 sport related PA levels in SCWT3, DTE, TMTA-B, and DTS. There were no significant differences between different levels of PA and dependent variables. Although the test did not show significance, SCWT showed a trend in $(\mathrm{p}=0.06)$ mean scores with vigorous group outperforming the low and moderate groups in the inhibition task of EF mean values for the low, moderate and vigorous group respectively $(116.65 \pm 27.37,100.63 \pm 25,98.83 \pm 29.11)$.

To see the differences between males and females in DTE, DTS, TMT-A, TMT-B and SCWT part 3 independent t-test was done. The test showed significant differences between STS, DTS and DTE for Males $(2.12 \pm 0.48(\mathrm{p}<0.02) ; 1.7 \pm 0.53$ $(\mathrm{p}<0.0005) ;-20.4 \pm 16.35(\mathrm{p}<0.01)$ compared to females, with mean values recorded substantially lower in STS, DTS, and $\operatorname{SCWT}(1.9 \pm 0.42,1.32 \pm 0.40,-29.86 \pm 19.56)$. Table 3 shows mean and standard deviation of EF, DT tests by gender. There were no significant differences in the rest of tests between males and females $p>0.05$. Our sample included ages between 18-40 grouped into group 1 (18 thru 24=1) $(25$ thru 34=2) (35 thru 40=3). One Way ANOVA was done to compare the means of the SCWT, TMT-A, TMT-B, DTS and DTE. Results showed significant differences in TMT-A, TMB and SCWT between the 3 groups respectively $(\mathrm{p}<0.001)$. While DTE, DTS, STS showed no difference. The Tukey post hoc test revealed that RA in age group 1 performed better in TMT-A $(18.36 \pm 6.46$ seconds, $\mathrm{p}<0.01)$ than group $2(23.2 \pm 9.33$ second, $\mathrm{p}<0.01)$ (Table 4).

\section{DISCUSSION}

Our study showed no significant differences in EF and DT in varying levels of sport related PA. However, a moderate trend was seen with the vigorous exercise group reporting higher scores in SCWT3 than other groups ( $\mathrm{p}=0.06$ ). Previous

Table 2. Descriptive values for all tests in mean \pm standard deviation

\begin{tabular}{lcccccc}
\hline IPAQ & TMT-A & TMT-B & SCWT-3 & STS & DTS & DTE \\
\hline Low & $19.64 \pm 6.18$ & $51.82 \pm 12.17$ & $116.65 \pm 27.37$ & $2.00 \pm 0.52$ & $1.46 \pm 0.4$ & $-25.5 \pm 19$ \\
Moderate & $20.16 \pm 5.80$ & $50.16 \pm 15.20$ & $100.63 \pm 25$ & $2.00 \pm 0.53$ & $1.53 \pm 0.6$ & $-24.4 \pm 17.94$ \\
Vigorous & $20.81 \pm 9.15$ & $50.32 \pm 19.55$ & $98.83 \pm 29.11$ & $2.06 \pm 0.45$ & $1.59 \pm 0.52$ & $-23.35 \pm 18.19$ \\
\hline
\end{tabular}

TMT-A-B: Trail Making Test A-B scores in seconds; SCWT3: Stroop Color Word Test scores in seconds; DTS-STS: Dual Task Speed-Single Task Speed in m/s; DTE: Dual Task Interference \%; IPAQ: International Physical Activity Questionnaire.

Table 3. Comparison of mean test scores among both genders

\begin{tabular}{lcrrrrc}
\hline Gender & TMT-A & \multicolumn{1}{c}{ TMT-B } & \multicolumn{1}{c}{ SCWT3 } & STS & DTS & DTE \\
\hline Male & $19.81 \pm 7.76$ & $49.72 \pm 16.90$ & $103.83 \pm 30.30$ & $2.12 \pm 0.47^{*}$ & $1.70 \pm 0.53^{* *}$ & $-20.39 \pm 16.35^{* * *}$ \\
Female & $21.78 \pm 8.93$ & $51.83 \pm 19.57$ & $97.29 \pm 24.83$ & $1.91 \pm 0.43^{*}$ & $1.32 \pm 0.40^{*}$ & $-29.89 \pm 19.56^{* * *}$ \\
\hline
\end{tabular}

TMT-A-B: Trail Making Test A-B scores in seconds; SCWT3: Stroop Color Word Test scores in seconds; DTS-STS: Dual Task Speed-Single Task Speed in m/s; DTE: Dual Task Interference \%.

Mean \pm standard deviation. ${ }^{*} \mathrm{p}<0.02,{ }^{*} \mathrm{p}<0.0005,{ }^{* * *} \mathrm{p}<0.01$.

Table 4. Comparison of mean test scores among age groups

\begin{tabular}{lcccccc}
\hline $\begin{array}{l}\text { Age groups } \\
\text { (years) }\end{array}$ & TMT-A & TMT-B & SCWT-3 & STS & DTS & DTE \\
\hline $18-24$ & $18.36 \pm 6.46^{*}$ & $44.73 \pm 13.47^{* *}$ & $92.80 \pm 22.22^{* *}$ & $2.02 \pm 0.45$ & $1.57 \pm 0.53$ & $-23.10 \pm 17.93$ \\
$25-34$ & $23.20 \pm 9.33^{*}$ & $56.35 \pm 21.84^{* *}$ & $112.27 \pm 32.4^{* *}$ & $2.08 \pm 0.56$ & $1.54 \pm 0.47$ & $-24.69 \pm 14.8$ \\
$35-40$ & $23.23 \pm 8.28^{*}$ & $65.50 \pm 15.57^{* *}$ & $114.20 \pm 28.35^{* *}$ & $2.03 \pm 0.34$ & $1.51 \pm 0.64$ & $-26.89 \pm 25.87$ \\
\hline
\end{tabular}

TMT-A-B: Trail Making Test A-B scores in seconds; SCWT3: Stroop Color Word Test scores in seconds; DTS-STS: Dual Task SpeedSingle Task Speed in $\mathrm{m} / \mathrm{s}$; DTE: Dual Task Interference $\%$.

Mean \pm standard deviation $* \mathrm{p}<0.001, * * \mathrm{p}<0.01$. 
research compared professional athletes participating in different sports or compared elites with RA. This is the first research studying comparing different sport types within the RA population, which may explain differences in results of previous research along with other factors that will be mentioned later on. EF has an integral part in sports physiotherapy, as shown $-n$ research reports of higher EF in athletes than nonathletes ${ }^{42}$. Specifically, elite athletes (team sports) reported higher EF than athletes ${ }^{43)}$. Another behavioral study, comparing inhibitory control in handball experts and RA, showed that experts outperformed RA in inhibitory control of foot and hands ${ }^{44)}$. Another study compared the differences in inhibition between professional and RA, results showed that professional cyclists performed more correct answers than the RA, furthermore, reflecting better inhibitory control ${ }^{45)}$. In RA, our results showed that there are no significant differences between EP and SP when it came to EF and DT, but that both groups fared equally well when compared to scores in the normal non-athletic population. This is different than previous research studies that identified differences between SP and EP sports between athletes. A meta -analysis reviewing the relationship between expertise in sports and laboratory-based measures of cognition showed that expertise SP types showed the largest statistically significant effect in processing speed than $\mathrm{EP}^{46)}$. Another study, with a small sample size, showed inhibitory control is greater for tennis players than swimmers and non-athletes, which is considered an open skill sport ${ }^{47)}$. However, previous research focused on sport expertise and elite athletes and may explain these differences due to sport-specific training in a or the intensity training experience that is different for RA. As mentioned before, training in a cognitive task may also enhance performance on related, but untrained, cognitive tasks ${ }^{55)}$. Another factor that may be different and yield different results from previous studies is prolonged training time, which may lead to adaptations in basic cognitive abilities causing performance differences between experts and novices even on tasks independent to the expert's domain ${ }^{54)}$.

It is worth mentioning, that no previous research has compared DT paradigms in healthy RA. Differences in cognitive performance between males and females were predicted by research studying the modal controllability between both genders $^{56)}$. Our study showed no differences between both genders in SCWT3, TMTA and B, but we also found that DTI for males was greater than female RA, which could be due to the fact that males had slightly faster dual and single task speed (STS) compared to females. When comparing TMT values by gender, age and educational level, it was shown that gender and age have an essential role on TMT which reflects processing speed and overlapping cognitive tasks, results showed that females outperform males ${ }^{48)}$ which was not the case in our findings. The authors speculate this may be due to the sedentary nature of the female population compared to male population recruited within the UAE. Gender differences in EF is not supported in the literature, as there are many other factors that may lead to such variations in EF between individuals and further study is needed to examine this issue within a cultural context. A longitudinal study conducted in India showed that early life education level plays a role in cognitive differences between females and males ${ }^{49}$ ) However, another study showed that tasks used to measure WM did not find any gender differences in developing sample from young children to older adult population ${ }^{50)}$. Considering age groups, a study found that the older the subjects the longer it took them to perform the test ${ }^{48)}$. Our results also showed that age groups (18-24) completed both tests TMTA-B and SCWT3 the fastest. However, there were no significant differences between age groups (25-34) and (35-40). Mean values were also similar between these two age groups. It can be interpreted as the peak inhibitory task, processing speed and other EF are greatest between age (18-24) years old. Middle age processing speed starts to decline which leads to decline in WM cognitive functions ${ }^{51)}$. Mean scores for all groups were within the average speed in TMTA-B which indicates that sports participation at any level would positively impact the processing speed and other cognitive function in RA. DTE between 3 groups did not differ which shows that exercise participation could have a positive effect on DT in RA, including older adults. It is mentioned previously that adults who are part of aerobic exercise would have better attentional control and EF than sedentary and non- aerobic exercises ${ }^{52,53)}$.

Hasher and Zacks highlighted the main sign of inhibitory age decline, which is the inability to focus on specific tasks while inhibiting the interference of information from previous scenarios requiring cognitive demand ${ }^{51)}$. In addition to previous findings, there were also cognitive function preservation among older adults with moderate to severe depression who were active. In this case moderate PA levels are required to exert a protective effect on mood and cognition in late adulthood ${ }^{24)}$. Limitations of our study include cross-sectional study design, as, unequal number of subjects in the three different sport related PA groups and small sample size. Future research should focus on measuring Recreational Sport related PA, including activity monitors throughout the week, and comparing DT paradigms in healthy athletic populations with those of a sedentary control group. Future research should also focus utilization of a longitudinal research design with equal participants among groups and larger sample size.

In conclusion, there were no significant differences in EF and DTE in RA, between the ages 18-40 years of age, between varying types of sport and level of sport related activity, though reported values were high across all groups. Males reported slightly better, but non-significant, DTE abilities than females in this study, which may be attributed to sedentary cultural expectations for females in the UAE. There was no significant difference in EF between males and females RA. EF performance was the highest among the age group (18-24) population, but in (25-34) and (35-40) had comparable results in EF and DTE. Our study concluded that multiple types and levels of participation in recreational sport activity are useful in maintaining high levels of EF performance for the RA population, between 18-40 years of age. Furthermore, SP activities may be an important alternative and conjunctive part of training for DT sport activities to train these skills. This research may also guide health care providers, including physiotherapists, to encourage the community to engage in any form of sports, to enhance DT and EF ability and prevent and decrease cognitive decline later on in life. 


\section{Funding}

The authors received no financial support for the research, or publication of this article.

Conflict of interest

The authors declare that there is no conflict of interest.

\section{REFERENCES}

1) Hughes C, Ensor R: Executive function and theory of mind in 2 year olds: a family affair? Dev Neuropsychol, 2005, 28: 645-668. [Medline] [CrossRef]

2) Wenner C, Bianchi J, Figueredo A, et al.: Life history theory and social deviance: the mediating role of executive function. Intelligence, 2013, 41: 41102-41113.

3) Collins A, Koechlin E: Reasoning, learning, and creativity: frontal lobe function and human decision-making. PLoS Biol, 2012, 10: e1001293. [Medline] [CrossRef]

4) Mennetrey C, Angeard N: Cognitive flexibility training in three-year-old children. Cogn Dev, 2018, 48: 125-134. [CrossRef]

5) Chevalier N: Les fonctions exécutives chez lenfant: concepts et développement. Can Psychol, 2010, 51: 149-163. [CrossRef]

6) Wideman TH, Asmundson GG, Smeets RJ, et al.: Rethinking the fear avoidance model: toward a multidimensional framework of pain-related disability. Pain, 2013, 154: 2262-2265. [Medline] [CrossRef]

7) Diamond A: Executive functions. Annu Rev Psychol, 2013, 64: 135-168. [Medline] [CrossRef

8) Robinson H, Calamia M, Gläscher J, et al.: Neuroanatomical correlates of executive functions: a neuropsychological approach using the EXAMINER battery. J Int Neuropsychol Soc, 2014, 20: 52-63. [Medline] [CrossRef]

9) Miyake A, Friedman NP, Emerson MJ, et al.: The unity and diversity of executive functions and their contributions to complex "Frontal Lobe" tasks: a latent variable analysis. Cognit Psychol, 2000, 41: 49-100. [Medline] [CrossRef]

10) Bryce D, Whitebread D, Szücs D: The relationships among executive functions, metacognitive skills and educational achievement in 5- and 7-year-old children. Metacognition Learn, 2014, 10: 181-198. [CrossRef]

11) Davidson RJ, Schwartz GE, Shapiro D: Consciousness and self-regulation. Boston: Springer, 1986.

12) Lundin-Olsson L, Nyberg L, Gustafson Y: "Stops walking when talking” as a predictor of falls in elderly people. Lancet, 1997, 349: 617. [Medline] [CrossRef]

13) Diamond A: Research that helps move us closer to a world where each child thrives. Res Hum Dev, 2015, 12: 288-294. [Medline] [CrossRef]

14) Verburgh L, Scherder EJ, van Lange PA, et al.: Executive functioning in highly talented soccer players. PLoS One, 2014, 9: e91254 [CrossRef]. [Medline]

15) Shields GS, Sazma MA, Yonelinas AP: The effects of acute stress on core executive functions: a meta-analysis and comparison with cortisol. Neurosci Biobehav Rev, 2016, 68: 651-668. [Medline] [CrossRef]

16) Smith E, Cusack T, Blake C: The effect of a dual task on gait speed in community dwelling older adults: a systematic review and meta-analysis. Gait Posture, 2016, 44: 250-258. [Medline] [CrossRef]

17) Chu YH, Tang PF, Peng YC, et al.: Meta-analysis of type and complexity of a secondary task during walking on the prediction of elderly falls. Geriatr Gerontol Int, 2013, 13: 289-297. [Medline] [CrossRef]

18) Al-Yahya E, Dawes H, Smith L, et al.: Cognitive motor interference while walking: a systematic review and meta-analysis. Neurosci Biobehav Rev, 2011, 35: 715-728. [Medline] [CrossRef]

19) Carone A: A compendium of neuropsychological tests: administration, norms, and commentary. Appl Neuropsychol, 2010, 14: 62-63. [CrossRef]

20) Ferro DA, Deijen JB, Koppes LL, et al.: The effects of physical activity and fitness in adolescence on cognition in adulthood and the role of insulin-like growth factor I. J Phys Act Health, 2016, 13: 392-402. [Medline] [CrossRef]

21) Bouchard C, Shephard RJ: Physical activity, fitness, and health: international proceedings and consensus statement. Champaign: Human Kinetics, 1994.

22) Barenberg J, Berse T, Dutke S: Executive functions in learning processes: do they benefit from physical activity? Educ Res Rev, 2011, 6: 208-222. [CrossRef]

23) Gold SM, Schulz KH, Hartmann S, et al.: Basal serum levels and reactivity of nerve growth factor and brain-derived neurotrophic factor to standardized acute exercise in multiple sclerosis and controls. J Neuroimmunol, 2003, 138: 99-105. [Medline] [CrossRef]

24) $\mathrm{Hu}$ L, Smith L, Imm KR, et al.: Physical activity modifies the association between depression and cognitive function in older adults. J Affect Disord, 2019, 246: 800-805. [Medline] [CrossRef]

25) Best JR: Exergaming immediately enhances children's executive function. Dev Psychol, 2012, 48: 1501-1510. [Medline] [CrossRef]

26) Jacobson J, Matthaeus L: Athletics, and executive functioning: how athletic participation and sport type correlate with cognitive performance. Psychol Sport Exerc, 2014, 15: 521-527. [CrossRef]

27) Tandon PS, Klein M, Saelens BE, et al.: Short term impact of physical activity vs. sedentary behavior on preschoolers' cognitive functions. Ment Health Phys Act, 2018, 15: 17-21. [CrossRef]

28) Dupuy O, Bosquet L, Fraser SA, et al.: Higher cardiovascular fitness level is associated to better cognitive dual-task performance in master athletes: mediation by cardiac autonomic control. Brain Cogn, 2018, 125: 127-134. [Medline] [CrossRef]

29) Oyeyemi AL, Oyeyemi AY, Adegoke BO, et al.: The Short International Physical Activity Questionnaire: cross-cultural adaptation, validation and reliability of the Hausa language version in Nigeria. BMC Med Res Methodol, 2011, 11: 156. [Medline] [CrossRef]

30) International Physical Activity Questionnaire-Short Form: https://journals.plos.org.

31) Craig CL, Marshall AL, Sjöström M, et al.: International physical activity questionnaire: 12-country reliability and validity. Med Sci Sports Exerc, 2003, 35: 1381-1395. [Medline] [CrossRef]

32) Sánchez-Cubillo I, Periáñez JA, Adrover-Roig D, et al.: Construct validity of the Trail Making Test: role of task-switching, working memory, inhibition/interference control, and visuomotor abilities. J Int Neuropsychol Soc, 2009, 15: 438-450. [Medline] [CrossRef]

33) Fellows RP, Dahmen J, Cook D, et al.: Multicomponent analysis of a digital Trail Making Test. Clin Neuropsychol, 2017, 31: 154-167. [Medline] [CrossRef] 
34) Zhou H, Sabbagh M, Wyman R, et al.: Instrumented trail-making task to differentiate persons with no cognitive impairment, amnestic mild cognitive impairment, and Alzheimer disease: a proof of concept study. Gerontology, 2017, 63: 189-200. [Medline] [CrossRef]

35) Scarpina F, Tagini S: The Stroop Color and Word Test. Front Psychol, 2017, 8: 557. [Medline] [CrossRef]

36) Doumas M, Rapp MA, Krampe RT: Working memory and postural control: adult age differences in potential for improvement, task priority, and dual tasking. J Gerontol B Psychol Sci Soc Sci, 2009, 64: 193-201. [Medline] [CrossRef]

37) Muhaidat J, Kerr A, Evans JJ, et al.: Validity of simple gait-related dual-task tests in predicting falls in community-dwelling older adults. Arch Phys Med Rehabil, 2014, 95: 58-64. [Medline] [CrossRef]

38) Plummer P, Eskes G: Measuring treatment effects on dual-task performance: a framework for research and clinical practice. Front Hum Neurosci, 2015, 9: 225 . [Medline] [CrossRef]

39) Kelly VE, Janke AA, Shumway-Cook A: Effects of instructed focus and task difficulty on concurrent walking and cognitive task performance in healthy young adults. Exp Brain Res, 2010, 207: 65-73. [Medline] [CrossRef]

40) Van Biesen D, Jacobs L, McCulloch K, et al.: Cognitive-motor dual-task ability of athletes with and without intellectual impairment. J Sports Sci, 2018, 36: 513-521. [Medline] [CrossRef]

41) Chen FT, Etnier JL, Wu CH, et al.: Dose-response relationship between exercise duration and executive function in older adults. J Clin Med, 2018, 7: 7. [Medline] [CrossRef]

42) Alves H, Voss MW, Boot WR, et al.: Perceptual-cognitive expertise in elite volleyball players. Front Psychol, 2013, 4: 36. [Medline] [CrossRef]

43) Cona G, Cavazzana A, Paoli A, et al.: It's a matter of mind! Cognitive functioning predicts the athletic performance in ultra-marathon runners. PLoS One, 2015, 10: e0132943. [Medline] [CrossRef]

44) Heppe H, Zentgraf K: Team handball experts outperform recreational athletes in hand and foot response inhibition: a behavioral study. Front Psychol, 2019, 10: 971. [Medline] [CrossRef]

45) Martin K, Staiano W, Menaspà P, et al.: Superior inhibitory control and resistance to mental fatigue in professional road cyclists. PLoS One, 2016,11 : e0159907. [Medline] [CrossRef]

46) Voss MW, Kramer AF, Basak C, et al.: Are expert athletes 'expert' in the cognitive laboratory? A meta-analytic review of cognition and sport expertise. Appl Cogn Psychol, 2009, 24: 812-826. [CrossRef]

47) Wang CH, Chang CC, Liang YM, et al.: Open vs. closed skill sports and the modulation of inhibitory control. PLoS One, 2013, 8: e55773. [Medline] [CrossRef]

48) Płotek W, Łyskawa W, Kluzik A, et al.: Evaluation of the Trail Making Test and interval timing as measures of cognition in healthy adults: comparisons by age, education, and gender. Med Sci Monit, 2014, 20: 173-181. [Medline] [CrossRef]

49) Lee J, Smith JP: Regional disparities in adult height, educational attainment and gender difference in late- life cognition: findings from the Longitudinal Aging Study in India (LASI). J Econ Ageing, 2014, 4: 26-34. [Medline] [CrossRef]

50) Grissom NM, Reyes TM: Let's call the whole thing off: evaluating gender and sex differences in executive function. Neuropsychopharmacology, 2019, 44: 86-96. [Medline] [CrossRef]

51) Hasher L, Zacks RT: Working memory, comprehension, and aging: a review and a new view. Psychol Learn Motiv, 1988, 22: 193-225. [CrossRef]

52) Albinet CT, Boucard G, Bouquet CA, et al.: Increased heart rate variability and executive performance after aerobic training in the elderly. Eur J Appl Physiol, 2010, 109: 617-624. [Medline] [CrossRef]

53) Kramer AF, Hahn S, Cohen NJ, et al.: Ageing, fitness and neurocognitive function. Nature, 1999, 400: 418-419. [Medline] [CrossRef]

54) Allen R, Fioratou E, McGeorge P: Cognitive adaptation: spatial memory or attentional processing. A comment on Furley and Memmert (2010). Percept Mot Skills, 2011, 112: 243-246. [Medline] [CrossRef]

55) Krenn B, Finkenzeller T, Würth S, et al.: Psychology of sport \& exercise sport type determines differences in executive functions in elite athletes. Psychol Sport Exerc, 2018, 38: 72-79. [CrossRef]

56) Cornblath EJ, Tang E, Baum GL, et al.: Sex differences in network controllability as a predictor of executive function in youth. Neuroimage, 2019, 188: 122-134. [Medline] [CrossRef]

57) IPAQ Web site: Guidelines for data processing and analysis of the International Physical Activity Questionnaire (IPAQ) — Short and Long Forms. http://www. ipaq.ki.se/dloads/IPAQ\%20LS\%20Scoring\%20Protocols_Nov05.pdf Published November 2005. (Accessed Jun. 2, 2020)

58) Trail Making Test (TMT) Parts A \& B. http://apps.usd.edu/coglab/schieber/psyc423/pdf/IowaTrailMaking.pdf. (Accessed Jun. 2, 2020) 